\title{
A smartphone application for educating the public about Alzheimer's disease and related dementias
}

\author{
Valerie Gruss PhD APN ANP-BC FAAN ${ }^{a, *}$, Memoona Hasnain MD PhD MHPE ${ }^{b}$ \\ ${ }^{a}$ Department of Biobehavioral Health Science, College of Nursing, University of Illinois at \\ Chicago, Chicago, Illinois, USA; ${ }^{b}$ Department of Family Medicine, College of Medicine, Uni- \\ versity of Illinois at Chicago, Chicago, Illinois, USA; *Corresponding author: vgruss@uic.edu
}

\begin{abstract}
Background: Though the prevalence of Alzheimer's disease and related dementias (ADRD) continues to rise, the majority of people feel uninformed and fearful of the disease. Mobile apps are a platform that can offer easy access to comprehensive information, advice, and resources. No apps are available that provide a coordinated, centralized source of information on ADRD, including practical advice to support persons with dementia and help them confront daily challenges throughout the disease continuum.

Objective: We examined the utility of a mobile app as a new approach to educating older adults with and without dementia, caregivers, and clinicians on how to navigate the challenging ADRD experience from diagnosis to end of life.

Methods: We developed a mobile app, Dementia Guide Expert, to deliver evidencebased and practical information about ADRD. We conducted initial usability testing of the app in older-age adults and practicing clinicians.

Results: Beta testing among older adults $(n=53)$ found that the content was engaging and useful but could be reorganized to improve access. Among clinicians $(n=20), 50 \%$ strongly agreed and $50 \%$ agreed that the app provided valuable information and practical advice and would be useful for people with ADRD and their caregivers. The app was launched in December 2017. As of March 2019, the app had been downloaded/viewed 24,353 times in 12 countries and it was recently translated into Spanish.

Conclusion: Using a mobile app to disseminate evidence-based information about ADRD is feasible and was well received by both older adults and clinicians. Plans are underway to translate the app into additional languages, which will help in global adoption, increase dementia awareness, and provide the world population with knowledge and skills to better understand and manage the challenges of ADRD.
\end{abstract}

Keywords: Dementia, application, geriatrics, smartphone, patient education

\section{INTRODUCTION}

According to the World Alzheimer's Report (2018), more than 50 million people worldwide have Alzheimer's disease or related dementias (ADRD)1, and this number is expected to nearly triple by 2050 . There are four main types of ADRDs: Alzheimer's Disease (AD; $(60-70 \%$ of dementias); Vascular Dementia (20\% of dementias); Dementia with Lewy Body (DLB; $5-10 \%$ of dementias); and Frontotemporal Dementia (FTD; $5 \%$ of dementias) (Types of Dementia/Alzheimer's Association, 2019).

As the most common dementia, AD affects 1 in every 10 people over age 65 in the US (Alzheimer's Disease Facts and Figures, 2018). ADRD is a chronic condition, lasting 8 to 10 years, during which the needs of the person with dementia (PwD) change and require different management and services with ongoing care. While there is no cure for ADRD, appropriate, holistic, and patientand family-centered care may slow the progres- sion of the disease and improve quality of life. Research indicates that PwD have a better quality of life, better survival, and lower resource utilization when there is improved care management in the community (Black et al., 2018). However, providing community-based care for PwD, including finding and accessing support resources and services, is a daunting task, and caring for PwD is a role for which most people are ill-prepared, but one they will likely have to play. A 2012 survey found that $75 \%$ of adults feel uninformed about $\mathrm{AD}$ and $44 \%$ fear it more than any other disease, including cancer (Business Wire, 2012).

Currently, access to ADRD information and caregiver support is fragmented and can come from various sources, including primary care physicians and national advocacy organizations, among others. Recognition of the need for ADRD education and support is reflected in the numerous calls to create infrastructure, programs, and policies to raise public awareness and provide information, 

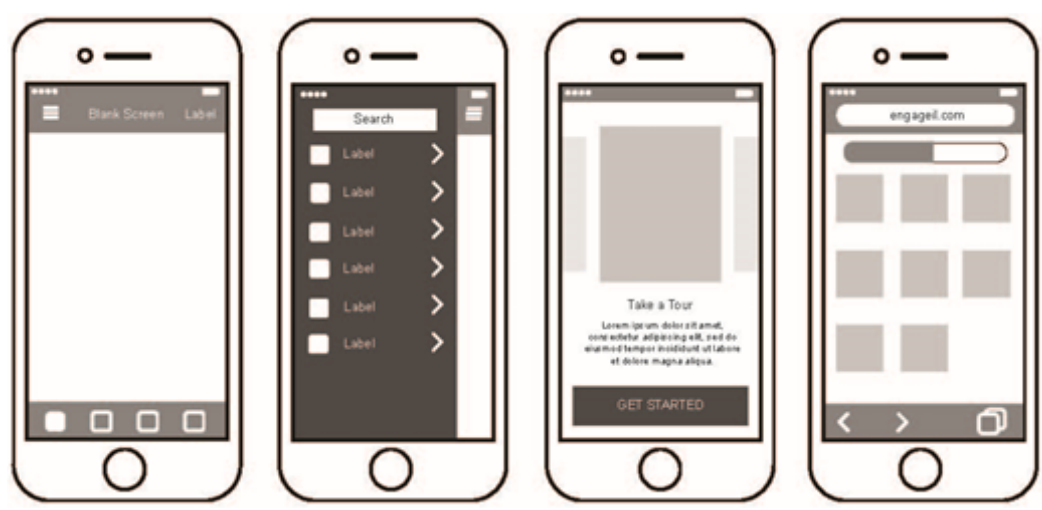

Figure 1. Wireframing (Google images) the Dementia Guide Expert app.

As our health care system moves from a focus on acute care settings to disease prevention and care management in the community, there is a need to provide solutions that support these new approaches. Mobile technology provides solutions to empower patients and facilitate them in achieving improved lifestyles, enabling patients to better

most notably the CDC's "Healthy Brain Initiative" (Centers for Disease Control and Prevention, 2018). Nearly all of these initiatives highlight the need for approaches to disseminate information about early detection and diagnosis. There remains a need for a coordinated, centralized, easily accessible source of information that will increase awareness and educate older adults with and without dementia, caregivers, and clinicians how to navigate through the challenging ADRD experience from diagnosis to end of life. Ideally, this information should include practical advice to support PwD and help them confront daily challenges throughout the disease continuum.

There is a proliferation of Mobile health ( $\mathrm{m}$-Health) technologies. In 2015, the Institute of Healthcare Informatics (IMS) (2015) reported 165,000 health apps were available for use among iOS and Android users. This explosion of mobile technology is driven by the ease of accessibility, afford-

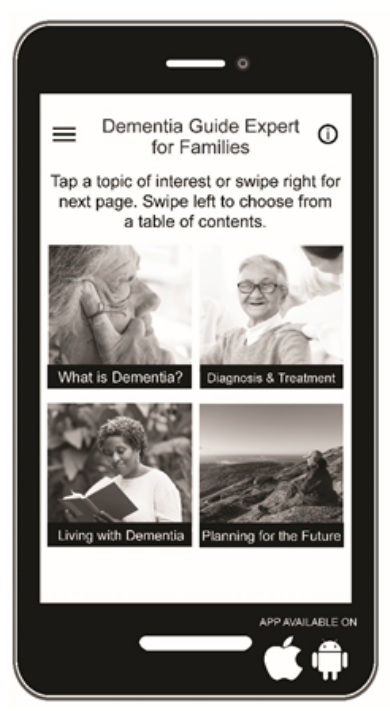

Figure 2. Dementia Guide Expert app Home Page design. self-manage their chronic diseases and provide enhanced remote access to treatments in difficult to reach communities.

The use of mobile health technologies is costeffective and accessible. Findings indicate mobile technology has been proven to enhance self-management of chronic diseases such as diabetes (de Ridder et al., 2017). Among older adult patients, $\mathrm{m}$-Health technologies have been shown to reduce emergency department visits by $70 \%$ and reduce hospital stays by $80 \%$ (Widmer et al., 2015).

Despite the proliferation of mobile app technology and the increase in health app usage, there are some challenges and limitations. These challenges include the lack of "evidence of clinical effectiveness" and "lack of integration within the healthcare delivery systems and the need for formal evaluation and review" (Centers for Disease Control and Prevention, 2018).

Recognizing the growing use of electronic tools and wireless technology in healthcare, we utilized care pathway technologies (Allen \& Rixson, 2018) to develop a downloadable mobile app, Dementia Guide Expert, for disseminating evidence-based information about ADRD and provide resources for PwD and caregivers. Cellphone ownership rates continue to increase, with $50 \%$ of persons over 50 years (Anderson, 2016) and $42 \%$ of persons over 65 years owning a smartphone (Anderson \& Perrin, 2016); thus, development of a mobile app was considered an optimal platform to deliver content on ADRD to the largest possible audience. In addition, research has found that accessing the internet by phone is similar among African Americans (98\%), Whites (94\%), and Hispanics (97\%) (Pew Research Center, 2018), allowing delivery of ADRD information in an unbiased manner. The purpose of this paper is to describe the development of the Dementia Guide Expert mobile app and the results of initial usability testing with older adults 
Visual/Observation Assessment:

$\square$ Ease of navigation (from screen to screen)

Readability (font, text size)

Usability of visual controls and icons

\section{Interview Questions:}

Functionality

Navigation:

Where the navigation arrows clear and easily understood?

Did you understand how to scroll, how to change views?

Did you know how to change out of "book view"?

Readability:

Was the font easy to read?

Did you know how to change font size?

Did the pictures provide visual cues?

\section{Usability}

Was it obvious if text was interactive vs. view only?

Was the "hamburger" menu intuitive?

Do you require a tutorial on how to use the app?

Figure 3. Beta testing: process for usability testing of the Dementia Guide Expert app in older-age adults.

and clinicians.

\section{Methods AND materials}

\section{App content development and navigation}

The objective of the Dementia Guide Expert app was to provide a comprehensive, centralized, easily accessible source of information about ADRD that spans the entire journey from diagnosis to end of life, with links to resources and support. The target audiences for the app were PwD, caregivers of PwD, older adults, and the general public. The app was developed through a collaborative partnership between content creators (geriatric clinicians at the University of Illinois Chicago [UIC]), the UIC Administrative

Table 1. Recommended changes to App based on Beta Testing User Observation and Interview of Older Adults ( $n=53$ )

\begin{tabular}{|c|c|}
\hline Domain & Comments/App Changes \\
\hline Navigation & $\begin{array}{l}\text { Make navigation arrows clear and consistent } \\
\text { Participants understood how to scroll but prefer if published studies list } \\
\text { was blinded } \\
\text { Provide links that jump user from summary information on a screen, to } \\
\text { a more detailed content below, on the same screen } \\
\text { Hamburger menu is not intuitive for all older adults }\end{array}$ \\
\hline Readability & $\begin{array}{l}\text { Participants request a glossary of icon meanings } \\
\text { Make it more obvious if text is interactive vs. view only } \\
\text { Define the non-pharmacological terms (e.g., Snoozem method) }\end{array}$ \\
\hline
\end{tabular}

Usability Create a short tutorial video with screen shots Rewrite information page

Provide a visual control that is very obvious on how to change font size Table of contents format was favored over large pictures to navigate Add link to TOC in white space below pictures

Make it more obvious how to get out of the "book" view

Change information button to HELP type icon
Information Technology Services (AITS), and intellectual property experts in the UIC Office of Technology Management. App development occurred over a period of 1 year using an iterative process.

App prototype content was created based on currently published literature and evidence-based guidelines for caring for persons with dementia and caregivers, with independent content review provided by the Alzheimer's Association. All app content was written in English for a broad audience at a 6th-grade reading level.

Content organization was considered concurrently with content creation using a storyboarding or "wireframing" process (Figure 1), which provided a visual guide for the app layout and the flow of information across various screens. Storyboards/wireframes were organized to establish functionality and the progress of users through each screen when navigating the app. We considered multiple screens flows based on the kinds of information users might search for on the app. A "home" button was added to each screen so that the user could return to the "home screen" at any time, as well as "next" and "back" buttons to allow users to navigate through the content screens. Users were not required to visit any particular screens and were allowed to access screens based on their individual needs.

Content for each app screen was created and organized to take users on a "journey" through the stages of dementia on the app. The content was organized into four sections: (1) What is dementia?, which provides an overview of ADRD, types and stages of dementia, and presenting signs and symptoms; (2) Diagnosis and Treatment, which describes DSM$\mathrm{V}$ (American Psychiatric Association, 2014) and NIA diagnostic guidelines (McKhann et al., 2011), pharmacologic treatment guidelines by the American Geriatrics Society (American Geriatric So- 


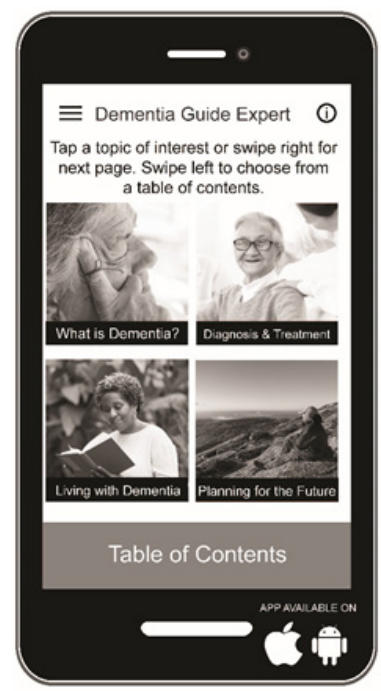

Figure 4. Dementia Guide Expert app with "Table of Contents" feature added. ciety, 2003) and American College of Physicians/American Academy of Family Physicians (Graham, 2008), and nonpharmacologic management approaches including psychosocial interventions, behavioral-orientated therapies, and cognitive therapies, based on published research (Dyer et al., 2018); (3) Living with Dementia, which provides guidance on managing behavioral symptoms, addressing changes in the environment and independence, how to create areas of safety to help PwD remain in the community and "age in place", and information about caregiver support; and (4) Planning for the Future, which provides information and resources on advance directives, end-of-life care, housing, and legal/financial resources. Citations to all articles were referenced in the content; however, to avoid cumbersome lists of references on screen, a tab was included on each screen that users could click to see all the literature/references for that screen. This tab could be closed to allow users to return to the content.

\section{App design and programming}

The design of the app was chosen to establish the visual appeal of the app and convey its purpose as

Please select the option that best reflects your opinion of the Dementia Guide Expert mobile app.

\begin{tabular}{lcccc}
\hline & $\begin{array}{c}\text { Strongly } \\
\text { Agree }\end{array}$ & Agree & Disagree & $\begin{array}{c}\text { Strongly } \\
\text { Disagree }\end{array}$ \\
\hline This app is easy to navigate & 0 & 0 & 0 & 0 \\
\hline The pictures enhance the experience & 0 & 0 & 0 & 0 \\
\hline The headings are large enough to read & 0 & 0 & 0 & 0 \\
\hline The text is large enough to read & 0 & 0 & 0 & 0 \\
\hline
\end{tabular}

Overall, the Dementia Guide Expert app...

\begin{tabular}{|c|c|c|c|c|}
\hline & $\begin{array}{l}\text { Strongly } \\
\text { Agree }\end{array}$ & Agree & Disagree & $\begin{array}{l}\text { Strongly } \\
\text { Disagree }\end{array}$ \\
\hline Provides valuable information & O & O & O & O \\
\hline Offers practical caregiving advice & 0 & 0 & O & 0 \\
\hline Supplies resources & O & 0 & O & 0 \\
\hline
\end{tabular}

Figure S1. Clinician online survey used for usability testing of the Dementia Guide Expert app with clinicians. a trusted, evidence-based source of information and resources for users. The design was based on previous experience developing the Behavioral Symptoms of Dementia app, (available for iOS and Android) with a home screen that used photo images to facilitate navigation (Figure 2).

The AITS group programmed the app using html coding and CSS3 for formatting, with the goal of producing two versions for use on a range of Android or Apple devices including phones, tablets, and iPads. Quality control testing was carried out by AITS and multiple prototypes were created. Dementia Guide Expert version 13 was submitted to Apple for a limited release for usability testing.

\section{Beta testing the app}

Internal testing was done continuously throughout the app development process to identify problems and make revisions. External testing (beta testing) was conducted to identify any user problems and obtain user feedback from two user segments from our targeted audiences: community-dwelling older adults and practicing clinicians who care for PwD.

First, we conducted a qualitative observational study to examine the app's usability and functionality among a convenience sample of independent, community-dwelling adults $>65$ years of age attending the local senior center. Eligible participants included all English-speaking older adults, age 65 and older, attending a local senior center. No person was excluded from this study based on their gender, race, or ethnicity or if they were experiencing memory loss. Eligible participants provided verbal consent prior to recruitment. Participants were provided an iPad with the app and allowed to use the app without direction for 10 minutes. During that time, participants were observed by the AITS app developers for ease of navigation, readability, and usability (Figure 3: Visual/Observation Assessment). Following the observation, the subjects continued to navigate for 10-20 minutes longer during which time they were interviewed by the team about navigation, readability, and usability of the app content (Figure 3: Interview Questions). The interview questions on navigation, readability, and usability were developed by the Office of Technology Management team.

For testing among selected practicing clinicians, Apple approved a limited online release of the app, called "Test Flight". To use TestFlight, we uploaded at least one beta build of the app to the App Store Connect and invited testers using their email addresses and by sharing a public link. Testers who accepted the invitation were instructed to follow the link to the app. Clinicians included physicians and nurse practition- 
Specifically, reflecting on the first section, "What is Dementia?"..

\begin{tabular}{|c|c|c|c|c|}
\hline & $\begin{array}{l}\text { Strongly } \\
\text { Agree }\end{array}$ & Agree & Disagree & $\begin{array}{l}\text { Strongly } \\
\text { Disagree }\end{array}$ \\
\hline Is useful for persons with dementia & 0 & 0 & 0 & 0 \\
\hline Is useful for caregivers & O & O & O & ( \\
\hline Provides valuable information & O & 0 & O & 0 \\
\hline Offers practical caregiving advice & ( & O & O & O \\
\hline Supplies resources & O & 0 & O & O \\
\hline
\end{tabular}

What information should be added to "What is Dementia?"

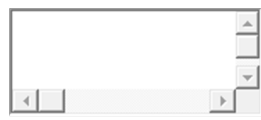

What are you drawn to in "What is Dementia?"

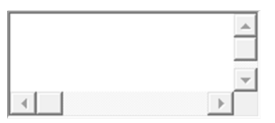

How can "What is Dementia?" be improved?

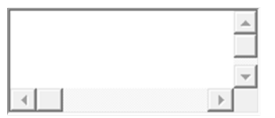

Figure S1. Clinician online survey used for usability testing of the Dementia Guide Expert app with clinicians (cont.).

ers working in primary care and geriatrics, in acute care, post-acute care, and outpatient clinic settings. Clinicians were asked to access the app, review the content, and complete an online sur-

Specifically, reflecting on the next section, "Living with Dementia"...

\begin{tabular}{lcccc}
\hline & $\begin{array}{c}\text { Strongly } \\
\text { Agree }\end{array}$ & Agree & Disagree & $\begin{array}{c}\text { Strongly } \\
\text { Disagree }\end{array}$ \\
\hline Is useful for persons with dementia & 0 & 0 & 0 & 0 \\
\hline Is useful for caregivers & 0 & 0 & 0 & 0 \\
\hline Provides valuable information & 0 & 0 & 0 & 0 \\
\hline Offers practical caregiving advice & 0 & 0 & 0 & 0 \\
\hline Supplies resources & 0 & 0 & 0 & 0 \\
\hline
\end{tabular}

What information should be added to "Living with Dementia"?

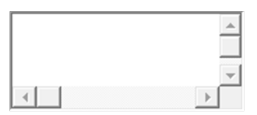

What are you drawn to in "Living with Dementia"?

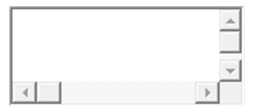

How can "Living with Dementia" be improved?

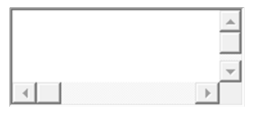

Figure S1. Clinician online survey used for usability testing of the Dementia Guide Expert app with clinicians (cont.). vey (Figure S1). The online survey was created by the authors and queried users about the value of the information, the practicality of the advice, the value of linked resources, overall usefulness, and app formatting.

\section{Results}

A total of 53 older adults consented to participate in beta testing. Participants "liked the pictures", found the content "important for everyone", "useful", and (to assist with navigation) recommended a "book style" format (Table 1). The app design was modified to include a table of contents and an option to access information through book-style chapters (Figure 4).

The app was released to 20 clinicians for beta testing. 50\% strongly agreed and 50\% agreed the app provided valuable information, offered practical advice, provided valuable resources, and was useful for people with dementia and caregivers. $67 \%$ agreed and 33\% strongly agreed the pictures enhanced their experience and the text was sufficiently large.

Initial usability and functionality testing with community-dwelling older adults and clinicians led to improvements in the organization and presentation of the content. The app was revised and prototype 14 was launched in December 2017, made available for free on iOS and Android devices. As of March 2019, the app had been downloaded/viewed 24,353 times in 12 countries (Australia, Brazil, Canada, China, Germany, Japan, Korea, New Zealand, Spain, Taiwan, UK, and USA).

\section{Discussion}

We developed a mobile app, Dementia Guide Expert, to deliver evidence-based ADRD education, including practical advice for persons living with dementia and links to caregiver resources and support. Initial usability testing with community-dwelling older adults and clinicians led to improvements in the organization and presentation of the content.

In 2017, there were 325,000 healthcare apps, ranging from fitness trackers to apps designed to help manage chronic conditions; these apps were downloaded approximately 3.7 billion times (Lastovetska, 2019). According to Modern Health Care (2013), there is a "convergence between devices and apps used by clinicians and those used by consumers". Consumer apps and devices are being integrated into comprehensive health care and wellness information system, with an increasing number of consumers accessing healthcare information through apps (Conn, 2013). A number of apps are available to help PwD; however, these apps are targeted 
Specifically, reflecting on the third section, "Diagnosis and Treatment"...

\begin{tabular}{|c|c|c|c|c|}
\hline & $\begin{array}{l}\text { Strongly } \\
\text { Agree }\end{array}$ & Agree & Disagree & $\begin{array}{l}\text { Strongly } \\
\text { Disagree }\end{array}$ \\
\hline Is useful for persons with dementia & 0 & 0 & 0 & 0 \\
\hline Is useful for caregivers & 0 & 0 & 0 & 0 \\
\hline Provides valuable information & 0 & 0 & 0 & 0 \\
\hline Offers practical caregiving advice & ○ & O & O & O \\
\hline Supplies resources & O & 0 & 0 & 0 \\
\hline
\end{tabular}

What information should be added to "Diagnosis and Treatment"?

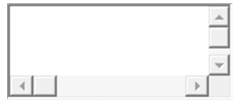

What are you drawn to in "Diagnosis and Treatment"?

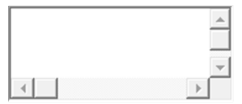

How can "Diagnosis and Treatment" be improved?

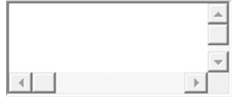

Figure S1. Clinician online survey used for usability testing of the Dementia Guide Expert app with clinicians (cont.).

to individuals already diagnosed, for example, apps for "memory games". The market lacked

Specifically, reflecting on the final section, "Planning for the Future"...

\begin{tabular}{lcccc}
\hline & $\begin{array}{c}\text { Strongly } \\
\text { Agree }\end{array}$ & Agree & Disagree & $\begin{array}{c}\text { Strongly } \\
\text { Disagree }\end{array}$ \\
\hline Is useful for persons with dementia & 0 & 0 & 0 & 0 \\
\hline Is useful for caregivers & 0 & 0 & 0 & 0 \\
\hline Provides valuable information & 0 & 0 & 0 & 0 \\
\hdashline Offers practical caregiving advice & 0 & 0 & 0 & 0 \\
\hline Supplies resources & 0 & 0 & 0 & 0 \\
\hline
\end{tabular}

What information should be added to "Planning for the Future"?

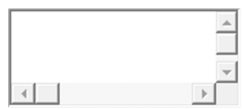

What are you drawn to in "Planning for the Future"?

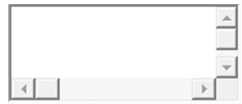

How can "Planning for the Future" be improved?

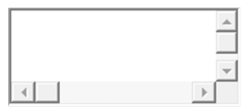

Would you recommend this app?

(1) Yes

(1) No

Figure S1. Clinician online survey used for usability testing of the Dementia Guide Expert app with clinicians (cont.). a centralized source of evidence-based, expertdeveloped information on appropriate management of ADRD, communication techniques, and links to resources and support services to assist PwD and caregivers as they journey through the trajectory of the illness, from diagnosis to end of life care. There was a clear unmet need for access to the comprehensive information and resources that we planned to include in the Dementia Guide Expert app.

The average direct cost of caring for a PwD in an institution is estimated to be threefold higher than in the community(Allegri et al., 2007; Beeri et al., 2002; Kraft et al., 2010). Given that health care resources are limited, there is a need for alternative models of care to allow PwD to "age in place", thereby reducing institutionalization and improving patient outcomes (Black et al., 2018). Research has focused on redesigning systems to link community resources, internet-based management protocols, and collaborative care planning with caregivers (Long et al., 2014). Integration of the Dementia Guide Expert into a comprehensive healthcare and wellness information system (Jahns, 2010) may help PwD age in place and improve the dementia experience by facilitating self-management of dementia symptoms (Toms et al., 2015) and providing links to relevant community resources to support PwD and caregivers. The Dementia Guide Expert may improve communication by helping PwD and caregivers ask informed questions and discuss specific concerns with clinicians at primary care visits and serve as a potential care coordination solution to manage dementia behavioral changes.

The app was recently translated into Spanish, "Guia Experta Sobre La Demencia". Plans are underway to translate the app into additional languages, which will help in global adoption, increasing dementia awareness and providing the world population with knowledge and skills to better understand and manage the challenges of ADRD. Further testing and refinement of the Dementia Guide Expert app are needed to evaluate its effectiveness in other target user groups, such as people with early-stage ADRD, caregivers, and the general public. User comments collected via iTunes and Google Play may also be used to further improve the content and usability of the app.

\section{Conclusions}

To create a "comprehensive healthcare and wellness information system" in the area of ADRD, we used mobile app technology to reach the largest audience possible to increase awareness and provide resources and support for caregivers. The dementia experience can be improved by helping older adults and caregivers access information and resources to help self-manage 
dementia symptoms. Educating the public about ADRD is likely to help create a society that is better prepared for the challenges of dementia care and encourage greater empathy toward PwD. Usability data indicate that Dementia Guide expert for families is being widely utilized in the United States and other countries. Although fur- ther research is warranted to evaluate the outcomes of our App, we believe we have made an important contribution to the health and wellbeing of PwDs and their caregivers by providing an evidence-based, comprehensive, technologybased, easily accessible and easy-to-use educational resource.

\section{References}

AGS Clinical Practice Committee. (2003). Guidelines abstracted from the American Academy of Neurology's Dementia Guidelines for Early Detection, Diagnosis, and Management of Dementia. J Am Geriatr Soc, 51(6), 869-873.

Allegri, R. F., Butman, J., Arizaga, R. L., Machnicki, G., Serrano, C., Taragano, F. E., Sarasola, D., Lon, L. (2007). Economic impact of dementia in developing countries: An evaluation of costs of Alzheimer-type dementia in Argentina. Int Psychogeriatr, 19(4), 705-718. https://doi.org/10.1017/ s1041610206003784

Allen, D., Rixson, L. (2008). How has the impact of 'care pathway technologies' on service integration in stroke care been measured and what is the strength of the evidence to support their effectiveness in this respect? Int J Evid Based Health, 6(1), 78-110. https://doi.org/10.1111/j.1744-1609.2007.00098.x

Alzheimer's Association. (2018). 2018 Alzheimer's disease facts and figures. Alzheimer's Dement, 14(3), 367-49. https://www.alz.org/media/HomeOffice/ Facts\%320and\%320Figures/facts-and- figures.pdf; retrieved March 320, 2019.

Alzheimer's Disease International. World Alzheimer's Report 2018. Retrieved March 22, 2019 from https://www.alz.co.uk/research/WorldAlzheimerReport2018.pdf

American Psychiatric Association. DSM-5 Coding Update. Supplement to Diagnostic and Statistical Manual of Mental Disorders, 5th ed., 2014. Retrieved March 20, 2019 from https://psychiatryonline.org/pb/assets/raw/dsm/pdf/DSM-5\%20Coding\%20Update_Final.pdf

Anderson, G. O. (2016). Technology trends among midlife and older Americans. AARP Research. 2016. Retrieved March 20, 2019 from https://www.aarp. org/research/topics/technology/info-2016/2016technology-trends-older-americans.html

Anderson, M., Perrin, A. (2017). Technology use among seniors. In: Tech Adoption Climbs Among Older Adults: Pew Research Center; 5-9. Retrieved March 20, 2019 from https://www.pewinternet. org/2017/05/17/technology-use-among-seniors/

Beeri, M. S., Werner, P., Adar, Z., Davidson, M., Noy, S. (2002). Economic cost of Alzheimer disease in Israel. Alzheimer Dis Assoc Disord, 16(2), 73-80.

Black, C. M., Fillit, H., Xie, L., Hu, X., Kariburyo, M. F., Ambegaonkar, B. M., Baser, O., Yuce, H., Khandker, R. K. (2018). Economic Burden, Mortality, and Institutionalization in Patients Newly Diagnosed with Alzheimer's Disease. J Alzheimers Dis, 61(1), 185-193. https://doi.org/10.3233/jad-170518

Centers for Disease Control and Prevention. Healthy Brain Initiative 2018. Retrieved March 20, 2019 from https://www.cdc.gov/aging/healthybrain/index.htm

Conn, J. (2013). No longer a novelty, medical apps are increasingly valuable to clinicians and patients. Mod Healthc, 43(50), 16-18, 20.

de Ridder, M., Kim, J., Jing, Y., Khadra, M., Nanan, R. (2017). A systematic review on incentive-driven mobile health technology: as used in diabetes management. Journal of Telemed Telecare;23(1):26-35. https://doi.org/10.1177/1357633X15625539

Devi, B. R., Syed-Abdul, S., Kumar, A., Iqbal, U., Nguyen, P., Li, Y. J., et al. (2015 Nov). mHealth: An updated systematic review with a focus on HIV/AIDS and tuberculosis long term management using mobile phones. Computer Methods and Programs in Biomedicine;122(2):257-265

Dyer, S. M., Harrison, S. L., Laver, K., Whitehead, C., Crotty, M. (2018). An overview of systematic reviews of pharmacological and non-pharmacological interventions for the treatment of behavioral and psychological symptoms of dementia. Int Psychogeriatr, 30(3), 295-309. https://doi.org/10.1017/ S1041610217002344

Graham, L. (2008). AAFP and ACP release guideline on dementia treatment. Am Fam Physician, 77(8), 1173-1175.

Home Instead. Americans Rank Alzheimer's as Most Feared Disease, According to New Marist Poll for Home Instead Senior Care. November 13, 2012. Retrieved March 20, 2019 from https://www. businesswire.com/news/home/20121113005422/ en/Americans-Rank-AIzheimer\%E2\%80\%99sFeared-Disease-New-Marist

IMS Institute for Healthcare Informatics. Availability and profile of consumer mHealth apps 2015. Retrieved on August 26, 2019 from https://www.iqvia.com

Jahns, R.-G. (2010). 500m people will be using healthcare mobile applications in 2015. Retrieved from https://research2guidance.com/500m-peoplewill-be-using-healthcare-mobile-applicationsin-2015-2/

Kraft, E., Marti, M., Werner, S., Sommer, H. (2010). Cost of dementia in Switzerland. Swiss Med Wkly, 140, w13093. https://doi.org/10.4414/ smw.2010.13093

Lastovetska, A. (January 24, 2019). Healthcare mobile app development: types, trends, \& features. MLSDev.. Retrieved March 20, 2019 from https://mlsdev.com/blog/why-develop-apps-for- healthcare

Long, K. H., Moriarty, J. P., Mittelman, M. S., Foldes, S. S. (2014). Estimating the potential cost savings from the New York University Caregiver Intervention in Minnesota. Health Aff (Millwood), 33(4), 596-604. https://doi.org/10.1377/hlthaff.2013.1257

McKhann, G. M., Knopman, D. S., Chertkow, H., Hyman, B. T., Jack, C. R., Jr., Kawas, C. H., Klunk, W. 


\section{Educating Alzheimer's disease and related dementias}

E., Koroshetz, WJ, Manly, JJ, Mayeux, R, Mohs, RC, Morris, JC, Rossor, MN, Scheltens, P, Carrillo, MC, Thies, B, Weintraub, S, Phelps, C. H. (2011). The diagnosis of dementia due to Alzheimer's disease: recommendations from the National Institute on Aging-Alzheimer's Association workgroups on diagnostic guidelines for Alzheimer's disease. Alzheimers Dement, 7(3), 263-269. https://doi. org/10.1016/j.jalz.2011.03.005

Pew Research Center. Mobile fact sheet. February 5, 2018. Retrieved March 20, 2019 from https://www. pewinternet.org/fact-sheet/mobile/

Toms, G. R., Quinn, C., Anderson, D. E., Clare, L. (2015). Help yourself: perspectives on self-man- agement from people with dementia and their caregivers. Qual Health Res25(1), 87-98. https://doi. org/10.1177/1049732314549604

Types of Dementia/Alzheimer's Association, 2019. Retrieved from https://www.alz.org/alzheimersdementia/what-is-dementia/types-of-dementia/ mixed-dementia

Widmer, R. J., Collins, N. M., Collins, C. S., West, C. P., Lerman, L. O., Lerman, A. (2015). Digital health interventions for the prevention of cardiovascular disease: a systematic review and meta-analysis. Mayo Clin Proc;90(4):469-480. https://doi.org/10.1016/j. mayocp.2014.12.026. 